\title{
Calcium and Vitamin $D$ increase mRNA levels for the growth control hIKI channel in human epidermal keratinocytes but functional channels are not observed
}

\author{
Vlasios Manaves ${ }^{1}$, Wuxuan Qin ${ }^{1}$, Amy L Bauer ${ }^{1}$, Sandra Rossie ${ }^{2}$, \\ Masakazu Kobayashi ${ }^{3}$ and Stanley G Rane*1,4
}

Address: ${ }^{1}$ Molecular Human Physiology Group, Fujisawa Research Institute of America, Evanston, IL 60201 USA, ${ }^{2}$ Department of Biochemistry, Purdue University, West Lafayette, IN 47907 USA, ${ }^{3}$ Fujisawa Research Institute of America, Evanston, IL 60201 USA and ${ }^{4}$ Depts. of Medicine, Evanston Northwestern Healthcare, Evanston, IL 60201, and Northwestern University Medical School, Chicago, IL 60611 USA

Email: Vlasios Manaves - val.manaves@fujisawa.com; Wuxuan Qin - wuxuan_qin@yahoo.com; Amy L Bauer - amy.bauer@fujisawa.com; Sandra Rossie - rossie@purdue.edu; Masakazu Kobayashi - masakazu.kobayashi@fujisawa.com; Stanley G Rane* - stanley.rane@fujisawa.com

* Corresponding author

Published: 16 June 2004

BMC Dermatology 2004, 4:7 doi:10.1 186/147/-5945-4-7
Received: 23 February 2004

Accepted: 16 June 2004

This article is available from: http://www.biomedcentral.com/I47I-5945/4/7

(C) 2004 Manaves et al; licensee BioMed Central Ltd. This is an Open Access article: verbatim copying and redistribution of this article are permitted in all media for any purpose, provided this notice is preserved along with the article's original URL.

\begin{abstract}
Background: Intermediate-conductance, calcium-activated potassium channels (IKs) modulate proliferation and differentiation in mesodermal cells by enhancing calcium influx, and they contribute to the physiology of fluid movement in certain epithelia. Previous reports suggest that IK channels stimulate proliferative growth in a keratinocyte cell line; however, because these channels indirectly promote calcium influx, a critically unique component of the keratinocyte differentiation program, an alternative hypothesis is that they would be antiproliferative and pro-differentiating. This study addresses these hypotheses.
\end{abstract}

Methods: Real-time PCR, patch clamp electrophysiology, and proliferation assays were used to determine if human IKI (hIKI) expression and function are correlated with either proliferation or differentiation in cultured human skin epidermal keratinocytes, and skin biopsies grown in explant culture.

Results: hIKI mRNA expression in human keratinocytes and skin was increased in response to anti-proliferative/ pro-differentiating stimuli (elevated calcium and Vitamin D). Correspondingly, the hIKI agonist I-EBIO inhibited keratinocyte proliferation suggesting that the channel could be anti-proliferative and pro-differentiating. However, this proliferative inhibition by I-EBIO was not reversed by a panel of hIKI blockers, calling into question the mechanism of I-EBIO action. Subsequent patch clamp electrophysiological analysis failed to detect hIKI channel currents in keratinocytes, even those expressing substantial hIKI mRNA in response to calcium and Vitamin D induced differentiation. Identical electrophysiological recording conditions were then used to observe robust IKI currents in fibroblasts which express IKI mRNA levels comparable to those of keratinocytes. Thus, the absence of observable hIKI currents in keratinocytes was not a function of the electrophysiological techniques.

Conclusion: Human keratinocyte differentiation is stimulated by calcium mobilization and influx, and differentiation stimuli coordinately upregulate mRNA levels of the calcium-activated hIKI channel. This upregulation is paradoxical in that functional hIKI channels are not observed in cultured keratinocytes. It appears, therefore, that hIKI does not contribute to the functional electrophysiology of primary human keratinocytes, nor intact human skin. Further, the results indicate caution is required when interpreting experiments utilizing pharmacological hIKI modulators in human keratinocytes. 


\section{Background}

Intermediate conductance, calcium-activated potassium channels (IKs) stimulate proliferative growth in T-cells and fibroblasts $[1,2]$, via their ability to maintain a negative membrane potential, thus enhancing the electromotive driving force for calcium entry [3], a mitogenic requirement in these cells. IKs have also been suggested to modulate secretion and cell volume in epithelial cells from the gut and airways [4-7], but it is not known if these channels also exert a growth control function in either isolated epithelial cells or intact epithelia. Unlike in T-cells and fibroblasts, stimulated calcium entry in human epidermal keratinocytes is associated with cessation (rather than enhancement) of proliferation, as well as promotion of the differentiation program [8]. Therefore, although across different cell types IKs may be expected to produce equivalent electrophysiological effects including the enhancement of calcium entry, in epithelial cells typified by keratinocytes this action may serve to limit proliferative activity and promote differentiation, the converse of IK function in T-cells and fibroblasts.

In support of a growth regulatory role for IK channels in human epidermal keratinocytes, the immortalized, nontumorigenic human keratinocyte cell line HaCaT has been shown to express human IK1 (hIK1) [9]. Interestingly, prolonged exposure of HaCaT to the IK activator, 1-EBIO, was reported to downregulate IK expression, an effect correlated with proliferative inhibition [10]. These results, although complicated by agonist induced channel expression changes, suggest a pro-proliferative role for IK in keratinocytes. Further, although potassium conductances have been reported in studies of primary, non-immortalized keratinocytes, it is unclear if these conductances are due to IK $[11,12]$. Thus, the exact physiological role of IK channels in primary human keratinocytes and in intact skin remains undetermined.

\section{Methods}

\section{Cell Culture}

Adult human epidermal keratinocytes (Cascade Biologics, Inc., Portland, OR or Cambrex Bio Science, Walkersville, $\mathrm{MD}$ ) were cultured per the supplier's instructions in EpiLife Medium with Human Keratinocyte Growth Supplement (bovine pituitary extract, $0.2 \% \mathrm{v} / \mathrm{v}$; bovine insulin, $5 \mu \mathrm{g} / \mathrm{ml}$; hydrocortisone, $0.18 \mu \mathrm{g} / \mathrm{ml}$; bovine transferrin, 5 $\mu \mathrm{g} / \mathrm{ml}$ and human epidermal growth factor, $0.2 \mathrm{ng} /$ $\mathrm{ml}$ (Cascade Biologics) in a $5 \% \mathrm{CO}_{2}, 37^{\circ} \mathrm{C}$ incubator. Basal calcium concentration was $0.06 \mathrm{mM}$. Stock cell cultures were passaged so as to maintain density at less than $70 \%$, and cultures were not used for more than six weeks after direct receipt from the supplier (typically within 510 passages). For gene expression and physiological studies keratinocytes were plated at densities of approximately $30-40 \%$ confluence at the start of the experiment.
Medium was then refreshed with or without various agents for chronic exposure experiments. Medium and additions were replenished every other day thereafter for the duration of the experiment.

\section{Cell proliferation and toxicity assays}

Keratinocytes were seeded in a 96-well plate at $10 \times 10^{3} /$ well in standard growth medium. After 2-4 hours (to allow attachment to substrate) test agents and $1 \mu \mathrm{Ci}{ }^{3} \mathrm{H}$ thymidine (Perkin Elmer Life Sciences/NEN Life Sciences, Boston, MA) were added. Approximately 24 hours later cells were harvested for scintillation counting of incorporated thymidine, expressed as CPM (each experimental group represents mean \pm SEM of triplicate samples). The CytoTox 96 Non-Radioactive Cytotoxicity Assay Kit (Promega, Madison, WI) was used to measure potential cytotoxic actions of test compounds.

\section{Electrophysiology}

Standard patch clamp techniques were used to attempt to record hIK1 currents in keratinocytes, and these procedures as applied to IK1 recordings from fibroblast cells have been extensively described ([13-15], and references therein). To facilitate patch seal formation and recording cells were rounded by brief (1-2 min) exposure to trypsin/EDTA, after which they were switched to the bath solution (in mM): $138 \mathrm{NaCl}, 9 \mathrm{KCl}, 1 \mathrm{CaCl}_{2}, 1 \mathrm{MgCl}_{2}$, and 10 HEPES. The patch pipette solution was $150 \mathrm{KCl}, 1$ $\mathrm{MgCl}_{2}, 10$ HEPES, with free calcium adjusted via addition of EGTA/calcium as described below. All solutions were at pH 7.4 and between 320-325 mOsm. Pipette resistances were between 2-4 M $\Omega$. Cells were held at $-70 \mathrm{mV}$ and stepped to $0 \mathrm{mV}$ (for $100 \mathrm{msec}$ ) in the presence of elevated intracellular calcium in order to activate hIK1 or mIK1 channels. Two approaches were used to elevate intracellular calcium to activate calcium-dependent currents. Either the free calcium concentration of the patch pipette solution was set to $10 \mu \mathrm{M}$ ( $1 \mathrm{mM}$ EGTA with $10 \mathrm{mM}$ calcium), or the pipette solution contained only $0.1 \mathrm{mM}$ EGTA (see above) and intracellular calcium was increased by extracellularly applying $10 \mu \mathrm{M}$ calcium ionophore (A23187) for 1-2 minutes. Compounds including A23187 were acutely applied to patch clamped cells by dissolving them in the bath solution, and then pressure ejecting the solution from blunt-tipped micropipettes positioned about 5$10 \mu \mathrm{M}$ from the cell. All recordings were performed at ambient temperatures $\left(21-23^{\circ} \mathrm{C}\right)$.

\section{Skin biopsies}

Skin biopsies (5 $\mathrm{mm}$ punches) were obtained from healthy donors via the Cooperative Human Tissue Network, Midwestern Division (Columbus, OH). For immediate RNA isolation biopsies were placed into RNALater (Ambion, Austin, TX) at the time of donation and shipped overnight in this solution. For experiments on living tis- 
sue biopsies were placed into EpiLife alone (with $1.4 \mathrm{mM}$ calcium) and shipped overnight on ice. Upon arrival in the lab living biopsies were trimmed of extraneous subdermal and dermal tissue, and were cultured in 6-well culture plates in EpiLife (with $1.4 \mathrm{mM}$ calcium) in a $5 \% \mathrm{CO}_{2}$, $37^{\circ} \mathrm{C}$ incubator. Medium with or without test agents was refreshed daily. All skin biopsies were obtained under procedures approved by the Institutional Review Board of the Evanston Northwestern Healthcare Research Institute.

\section{RNA Isolation}

Total RNA was extracted from cultured human keratinocytes and skin biopsies with the RNAqueous phenol-free RNA isolation kit (Ambion, Austin, TX). RNAlater (Ambion) preserved skin samples were homogenized in RNAqueous lysis buffer with an IKA Works Ultra-Turrax T8 dispersing instrument (IKA Works, Wilmington, NC). Cell debris were removed from the homogenates by centrifuging for two to three minutes at $12,000 \mathrm{~g}$. RNA was subsequently purified according to manufacturer's instructions. Keratinocytes were washed twice with Dulbecco's Phosphate Buffered Saline then homogenized with lysis buffer directly in culture dishes using a cell scraper. Keratinocyte RNA was purified as per instructions of manufacturer. RNA concentrations were determined by measuring absorbance at $260 \mathrm{~nm}$. Ratios of absorbance at 260/280 nm yielded values between 1.8 and 2.1. All samples were DNase treated for at least $1 \mathrm{hr}$ prior to reverse transcription.

\section{RT-PCR}

cDNA was synthesized by reverse transcribing $0.5-2 \mu \mathrm{g}$ of total RNA using Power Script reverse transcriptase (BD Biosciences Clontech, Palo Alto, CA) in a $40 \mu \mathrm{L}$ reaction. Briefly, samples were pre-incubated at $70^{\circ} \mathrm{C}$ with $20 \mathrm{ng}$ of random decamers (Ambion) for two minutes, cooled on ice then incubated at $42^{\circ} \mathrm{C}$ for 90 minutes. Reactions were terminated with a final $15^{\circ} \mathrm{C}$ incubation for 15 minutes.

Expression levels of the targeted mRNA were quantified by fluorescent real-time polymerase chain reaction using an ABI Prism 7700 Sequence Detector (Applied Biosystems, Foster City, CA). Real-time PCR reactions were performed in triplicate in a total volume of $30 \mu \mathrm{L}$ containing $1 \mu \mathrm{L}$ of cDNA, $15 \mu \mathrm{L}$ of Universal PCR Master Mix (Applied Biosystems), 300-900 nM of each forward and reverse primer and $200 \mathrm{nM}$ of each probe. Gene specific Taq-Man primers and probes were designed and synthesized using the Primer Express software package (Applied Biosystems). Primer and probe sequences for hIK1 were (5'-3'): GTGAACTCCATGGTGGACATCTC (forward), CGGTGTGAGCTGCTCAGATTC (reverse), ATGCACATGATCCTGTATGACCTGCAGC (probe); and, for mIK1: TGCCTTCCTCCTTTGTCTTATTGT (forward), AGCCCGTTGTCAGTCATGAAC (reverse), TCTTCCAT-
GCCAAGGAGGTCCAGC (probe). Probes were labeled with the fluorescence reporter FAM at the $5^{\prime}$ end, and the fluorescence quencher TAMRA at the 3 ' end. GAPDH was detected using primers and a VIC labeled probe purchased from Applied Biosystems as a Pre-developed Taq-Man Assay Reagent. PCR reaction conditions were as follows: two minutes at $50^{\circ} \mathrm{C}$ to activate uracil-N-glycosylase (Amp-Erase UNG) $95^{\circ} \mathrm{C}$ for 5 minutes to deactivate UNG, 40 cycles at $95^{\circ} \mathrm{C}$ for 15 seconds and $60^{\circ} \mathrm{C}$ for one minute. The relative quantification of mRNA, described as either fold change from control or \% of GAPDH, was determined by the comparative $\mathrm{Ct}$ method where the target is normalized to the endogenous reference GAPDH. The threshold $\mathrm{Ct}$ value is the cycle number arbitrarily selected from the logarithmic phase of the PCR curve where an increase in fluorescence can be detected above background. The $\Delta \mathrm{Ct}$ is determined by subtracting the $\mathrm{Ct}$ of GAPDH from the Ct of the target $(\Delta \mathrm{Ct}=\mathrm{Ct}$-target $-\mathrm{Ct}$ $\mathrm{GAPDH}$ ). The relative value of target to endogenous reference is described as $\%$ of GAPDH $=2^{-\Delta \mathrm{Ct}}$. Fold change from control $=2^{-\Delta \Delta \mathrm{Ct}}$ where $\Delta \Delta \mathrm{Ct}=\Delta \mathrm{Ct}$-control $-\Delta \mathrm{Ct}$ treatment.

To perform end-point PCR for 1281 bp of the $1284 \mathrm{bp}$ coding sequence of hIK1 PCR reactions were run in a reaction volume of $50 \mu \mathrm{L}$ using Advantage-GC 2 Polymerase with $15 \mu \mathrm{L}$ of GC melt (BD Biosciences Clontech, Palo Alto, CA), 1X GC 2 PCR Buffer, $200 \mu \mathrm{M}$ dNTP, $0.4 \mu \mathrm{M}$ of each primer and $5 \mu \mathrm{L}$ of cDNA. PCR reaction parameters were as follows: $94^{\circ} \mathrm{C}$ for 3 minutes, 35 cycles of $94^{\circ} \mathrm{C}$ for 15 seconds and $68^{\circ} \mathrm{C} 3$ minutes, and a final extension at $68^{\circ} \mathrm{C}$ for 3 minutes. PCR for the amplification of GAPDH mRNA was performed in the same manner except 30 cycles were used and the GC melt was omitted. Primer sequences for hIK1 were (5'-3'): ATGGGCGGGGATCTGGTGCTT (forward), CTTGGACTGCTGGCTGGGTTC (reverse). GAPDH primers (Maxim Biotech, Inc., San Francisco, CA) were (5'-3'): TGCTGGCGCTGAGTACGTCGT (forward), GTGGAGGAGTGGGTGTCGCTG (reverse) yielding a PCR product of $615 \mathrm{bp}$. PCR products were analyzed on a $1.5 \%$ agarose gel stained with ethidium bromide.

\section{Reagents and chemicals}

The following compounds were prepared as stocks for application to cell cultures or single cells after appropriate dilution in either culture medium or physiological saline, respectively. VitD (calcitriol; 1,25- $(\mathrm{OH})_{2}-\mathrm{D}_{3}$, Sigma Corp., St. Louis, MO) in DMSO; A23187 in DMSO (Sigma); $50 \mathrm{mM}$ 1-EBIO (1-ethyl-2-benzimidazolinone, Tocris Cookson Inc., Ellisville, MO) in ethanol; $25 \mathrm{mM}$ clotrimazole (Sigma) in ethanol; $10 \mu \mathrm{M}$ charybdotoxin (Tocris) in $10 \mathrm{mM}$ acetic acid with $0.1 \%$ BSA; $1 \mathrm{mM}$ TRAM-34 in DMSO. TRAM-34 was a generous gift of Dr. Heike Wulff (UC Davis School of Medicine). Correspond- 


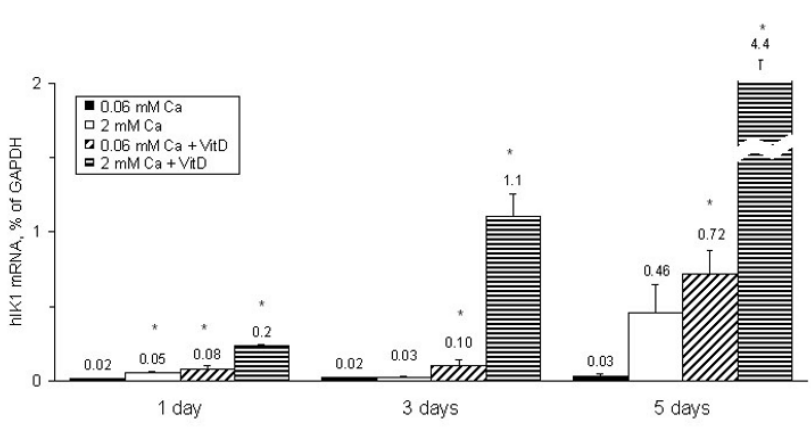

Figure I

hIKI mRNA expression is upregulated in human epidermal keratinocytes under culture conditions which are anti-proliferative/pro-differentiating. Keratinocytes were grown in either proliferative $(0.06 \mathrm{mM}$ calcium), or anti-proliferative, pro-differentiating culture conditions (2 mM calcium, I $\mu M$ VitD, or both) for I, 3, and 5 days. Total mRNA was harvested and processed for real-time PCR analysis. hIKI mRNA levels are expressed as \% of GAPDH mRNA, with data values at the top of each respective column. Each column represents the mean \pm SEM for 2 to 6 cultures treated as indicated. The far right data column is broken to allow scaling of the $y$-axis appropriate to the lower expression values. * indicates significant difference from 0.06 $\mathrm{mM}$ calcium treatment at the indicated number of days.

ing vehicle controls showed no effect on cell proliferation, cell toxicity, electrophysiology, or gene expression.

\section{Data Analysis}

Numerical results are given as mean \pm SEM unless otherwise noted. One-way ANOVA was used for comparisons of treated groups to a control no-treatment group, as well as for pairwise comparisons among treatment groups. The $95 \%$ confidence interval was used to assess significance for these analyses.

\section{Results and Discussion hIKI mRNA expression in proliferating and differentiating human keratinocytes}

We examined whether or not hIK1 mRNA is expressed in primary human epidermal keratinocytes, and if this expression is altered by addition of VitD at $1 \mu \mathrm{M}$, a concentration which is maximal for anti-proliferative/pro-differentiating effects [16], or elevation of extracellular calcium from 0.06 to $2.0 \mathrm{mM}$. Both of these perturbations induce expression of differentiation genes, with elevated calcium also producing profound morphological changes consistent with the onset of the differentiation program $[17,18]$. Both elevated calcium and VitD significantly increased hIK1 mRNA levels within 24 hours and these increases were maintained over the course of five days exposure (figure 1). The combination of VitD and elevated calcium produced synergistic increases in hIK1 expression at days 3 and 5 . These results are consistent with the hypothesis that in human keratinocytes hIK1 may be both anti-proliferative and a positive regulator of the differentiation process.

The effect of VitD on hIK1 levels was also assessed in cells that had been previously exposed to elevated extracellular calcium, simulating what might occur in intact skin when VitD is therapeutically applied in part because of its prodifferentiating properties. In this experiment hIK1 expression in basal calcium $(0.06 \mathrm{mM})$ was $0.029 \%$ of GAPDH, and after 48 hours in $2 \mathrm{mM}$ calcium the value was $0.068 \%$, similar to the result shown in figure 1 . In cells that were treated with $2 \mathrm{mM}$ calcium for 24 hours followed by addition of $1 \mu \mathrm{M}$ VitD for another 24 hours hIK1 expression was $0.165 \%$, again comparable to results shown in figure 1 for 24 hour calcium and VitD exposure. GAPDH levels, which were used to normalize gene expression, varied little in response to calcium and/or VitD (GAPDH Ct difference between untreated and treated cells of $0.2 \pm 0.2, \mathrm{~N}=9$ determinations), further substantiating the robustness of the hIK1 upregulation results.

\section{hIKI mRNA expression in human skin biopsies}

To directly assess the significance of the cell culture results to intact skin, hIK1 mRNA was assayed in human skin biopsies. To assess the variability of baseline hIK1 expression among skin samples, sets of three skin punch biopsies from each of three donors were placed in RNALater at the donation site. hIK1 mRNA expression in these biopsies ranged from 0.10 to $0.21 \%$ of GAPDH expression, with the values being quite consistent among biopsies from a given donor as well as between donors (figure 2A). Skin hIK1 expression values were somewhat higher than those observed in cultured keratinocytes growing under basal conditions $(0.02 \%$ of GAPDH, $0.06 \mathrm{mM}$ calcium no added VitD). However, keratinocytes treated with VitD alone or calcium and VitD expressed hIK1 at levels comparable to those in skin. mRNA levels for involucrin (INV) and transglutaminase-1 (TG-1), two contributors to and markers of keratinocyte differentiation, also showed little inter or intradonor variability. Expression levels for $\operatorname{INV}(9.4 \pm 1.6 \%$ of GAPDH, $\mathrm{N}=9)$ and TG-1 $(2.7 \pm 0.4 \%$, $\mathrm{N}=9$ ) in the biopsies were higher than levels observed in untreated cultured keratinocytes (INV $4.9 \pm 0.7 \%$, TG-1 $0.74 \pm 0.19 \%)$. However, treatment of cultured keratinocytes with calcium, VitD or both agents elevated levels of INV (3.1-fold) and TG-1 (2.5-fold) into the ranges observed for skin biopsies.

To determine the dynamics of hIK1 expression, hIK1 expression levels were measured as a function of time after biopsy collection and subsequent growth in explant cul- 

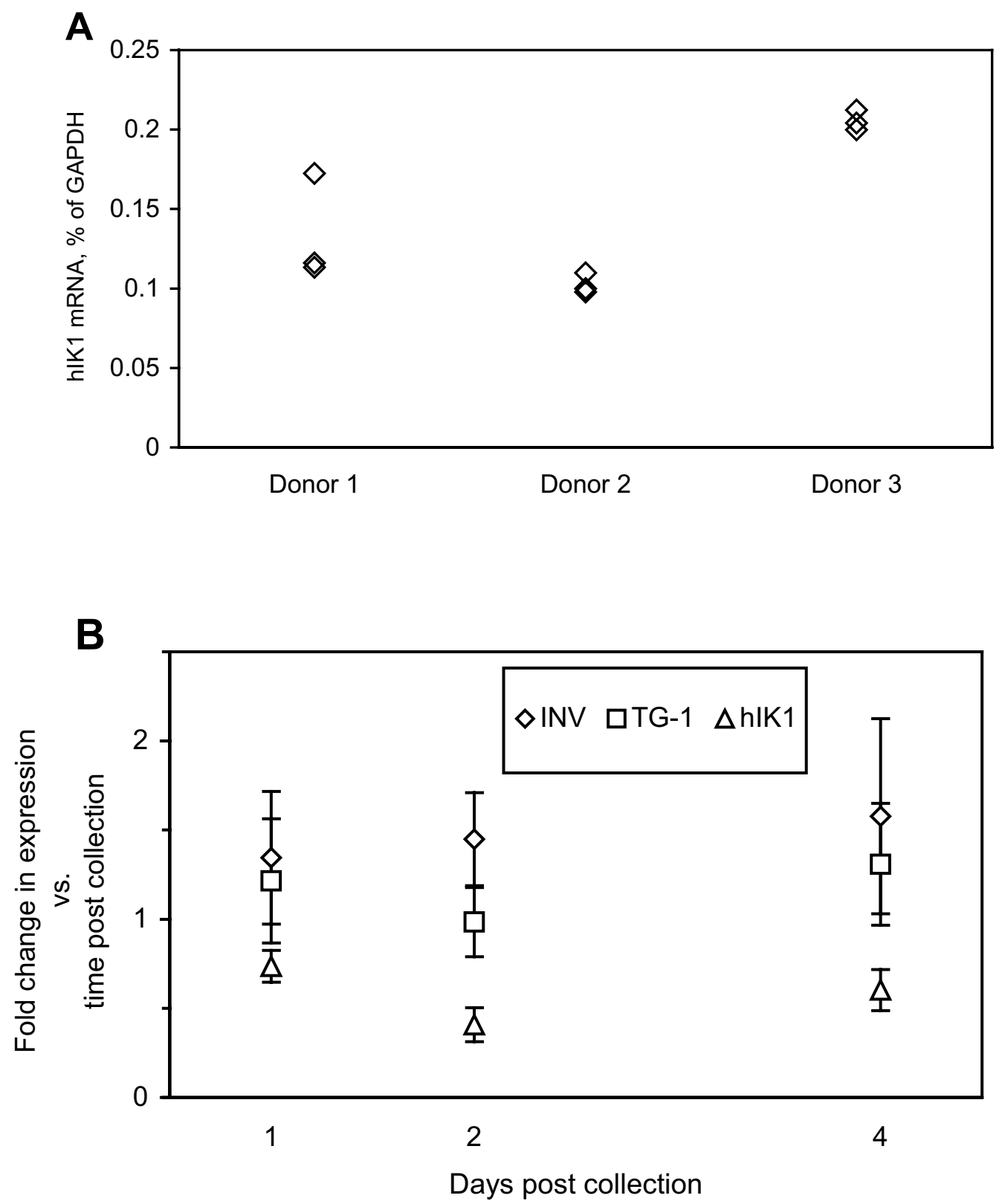

\section{Figure 2}

Consistent hIKI mRNA levels among biopsies from individuals and donor groups are maintained in explant culture. Real-time PCR analysis of mRNA expression levels (normalized to GAPDH) in human skin biopsies from healthy donors. (a) data points are hIKI mRNA levels from three biopsies from each of three donors with mRNA harvesting performed at time of biopsy collection. There were no statistically significance differences in hIKI expression among the three donors. (b) effects of culture time on gene expression in explant biopsies assessed by measuring mRNA levels from biopsies preserved in RNALater at time of collection, and assessing fold changes from these levels in sibling biopsies shipped overnight in culture medium ( $I$ day post collect), or shipped and kept in culture for I and 2 additional days ( 2 and 4 days post collect). Expression of involucrin (INV) and transglutaminase (TG-I) are shown for comparison with hIKI. Each data point is mean \pm SEM for 3 biopsies. For any given gene there were no statistically significant differences in expression when comparing data from days one, two, and four. 


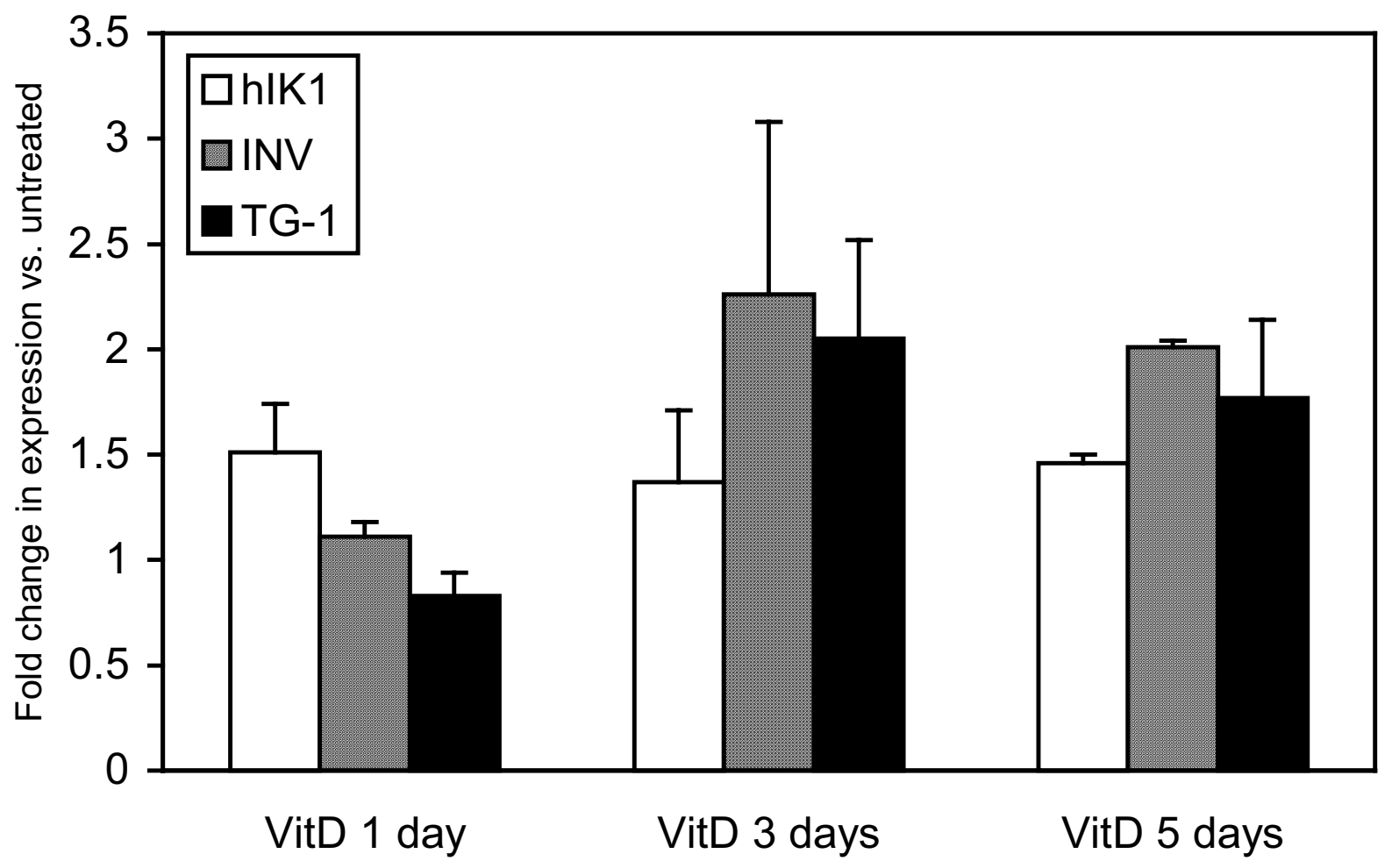

Figure 3

VitD modestly upregulates mRNA for hIKI (and INV, TG-I) in explant cultured skin biopsies. Real-time PCR analysis of mRNA expression levels (normalized to GAPDH) in human skin biopsies from healthy donors. Each column represents the mean \pm SEM fold change in mRNA levels for three treated biopsies relative to three untreated, time-matched controls. Changes in gene expression did not reach statistical significance.

ture (figure 2B). Medium always contained $1.4 \mathrm{mM}$ calcium, a requirement for survival of explant skin cultures [19]. At 2 days post collection ( 1 day shipping, 1 day in culture) hIK1 expression had decreased to $40 \%$ of its initial value, but with an additional 2 days in culture expression had recovered to $60 \%$ of its initial value. TG-1 expression showed a similar decrease followed by an increase with culture time, while INV increased insignificantly. Skin biopsies contain dermal fibroblasts that may express hIK1. Although we trimmed much of the dermal tissue from the biopsies used for this study, we cannot completely discount some contribution of dermal fibroblasts to the hIK1 expression results.

We examined the effect of VitD exposure over time on skin explant hIK1 expression. In response to $1 \mu \mathrm{M}$ VitD in the culture medium hIK1 expression increased slightly to approximately 1.5 -fold of the untreated condition, and remained elevated for the duration of the experiment (figure 3). INV and TG-1 expression remained relatively unchanged at day one, followed by approximately 2 -fold increases at days three and five. Thus the ability of VitD to enhance hIK1 mRNA expression in explant biopsies was less than for cultured keratinocytes.

\section{Effect of the hIKI activator I-EBIO on keratinocyte proliferation}

The increase in hIK1 expression under anti-proliferative/ pro-differentiating growth conditions (calcium, VitD) was surprising in that increased IK channel expression has typically been correlated with proliferative stimuli and growth $[1,2]$. In addition, contact induced proliferative inhibition in the HaCaT keratinocyte line correlated with decreased hIK1 expression [9], and pharmacological downregulation of hIK1 correlated with mitogenic inhibition [10]. However, differentiation marker expression did 
not increase, and it must be noted that proliferative inhibition is not necessarily coupled to enhanced cell differentiation. Our results correlating increased hIK1 expression with keratinocyte differentiation are, however, consistent with the general physiological model for this channel's function, namely to enhance calcium influx. Thus, in keratinocytes where calcium influx is a differentiation signal, hIK1 would be predicted to have anti-proliferative and/or pro-differentiating functions.

To more directly test this hypothesis that hIK1 activation would be inhibitory towards keratinocyte proliferation, we looked at the effect of the hIK1 activator 1-EBIO on tritiated thymidine incorporation, a measure of mitogenic activity. Indeed, 1-EBIO inhibited mitogenesis with an apparent $\mathrm{IC}_{50}$ slightly greater than $100 \mu \mathrm{M}$ (figure 4), close to the $\mathrm{EC}_{50}$ for 1-EBIO activation of the cloned hIK1 channel $(74-84 \mu \mathrm{M},[20,21])$. This effect was not due to 1 EBIO induced cytotoxicity, as an LDH release based assay showed no cytotoxicity up to $300 \mu \mathrm{M}$ 1-EBIO (data not shown). Surprisingly, however, mitogenic inhibition due to 1-EBIO could not be reversed by any of a panel of hIK1 blockers, including ChTX $(0.2 \mu \mathrm{M})$, clotrimazole $(0.3$ $\mu \mathrm{M})$, and TRAM-34 (0.1 and $1 \mu \mathrm{M})[1,2,22]$. The blockers themselves did not inhibit mitogenesis except for a modest effect of TRAM-34 ( $<30 \%$ inhibition) at the relatively high concentration of $1 \mu \mathrm{M}\left(400-500\right.$ times the $\mathrm{IC}_{50}$ for IK block). The inability of these blockers to reverse mitogenic inhibition by 1-EBIO called into question whether hIK1 was indeed the target of 1-EBIO's anti-proliferative action. Thus, these results required a functional examination of hIK1 activity in primary keratinocytes.

\section{Electrophysiological evaluation of hIKI functional expression}

To directly correlate expression and pharmacological data with hIK1 channel function, we attempted to record hIK1 currents in keratinocytes. Whole cell patch clamp recordings were performed on a total of 41 keratinocytes. Only recordings with seal resistances greater than 5 G $\Omega$ were deemed acceptable for data acquisition. The number of cells recorded for each treatment condition, and the ranges of cell capacitances were as follows: 4 untreated, $12-19 \mathrm{pF} ; 4$ at $2 \mathrm{mM}$ calcium for 3 days, $18-35 \mathrm{pF} ; 14$ at $1 \mu \mathrm{M}$ VitD for 3 days, $14-40 \mathrm{pF} ; 11$ at $1 \mu \mathrm{M}$ VitD for 5 days, $27-58 \mathrm{pF} ; 5$ at $1 \mu \mathrm{M}$ VitD and $2 \mathrm{mM}$ calcium for 3 days, 34-62 pF; 3 at $1 \mu \mathrm{M}$ VitD and $2 \mathrm{mM}$ calcium for 5 days 45-61 pF. The increase in cell capacitances (i.e., cell area) in response to the differentiating agents calcium and VitD is consistent with the increase in cell size observed when cells withdraw from the cell cycle and differentiate. Intracellular calcium in cells was increased via either the patch pipette solution ( $10 \mu \mathrm{M}$ free calcium) or by external application of $10 \mu \mathrm{M}$ A23187 for 1-2 minutes, but hIK1 currents, as evidenced by increased outward currents at 0
$\mathrm{mV}$, were not observed under either condition (figure 5). In 17 cells challenged with A23187, current amplitudes at -70 and $0 \mathrm{mV}$ were $-42.3 \pm 32.2$ and $-3.2 \pm 5.6 \mathrm{pA}$ respectively before challenge, and $-48.3 \pm 29.2$ and $-4.4 \pm 5.7 \mathrm{pA}$ after. An additional 21 cells exposed to $10 \mu \mathrm{M}$ free calcium via the patch pipette had current amplitudes of -54.1 $\pm 45.2 \mathrm{pA}$ and $-1.5 \pm 6.6 \mathrm{pA}$ at -70 and $0 \mathrm{mV}$ respectively, again showing no significant calcium induced current increase at $0 \mathrm{mV}$ as would be expected for IK activation. Three of the cells in the 5 day VitD group were also simultaneously exposed to the IK agonist 1-EBIO $(100 \mu \mathrm{M})$ and $10 \mu \mathrm{M}$ internal free calcium, but again no hIK1 currents were evoked (current amplitude at $0 \mathrm{mV}$ of $-2.0 \pm 4.2 \mathrm{pA}$ ). Inside-out patches also were made from 3 additional cells treated with VitD and calcium for 5 days, but no hIK1 channel activity was observed in these patches. We found no evidence of voltage-gated currents in keratinocytes at any test potentials investigated from -40 to $80 \mathrm{mV}$ (data not shown).

To assure that the recording conditions were satisfactory for detecting hIK1 activity, we used the same solutions and reagents to evoke mouse IK1 (mIK1) currents in rastransformed NIH3T3 cells (figure 5). Currents due to mIK1, a homolog of hIK1 [23], were detected in all four cells tested, with current amplitudes ranging from $0.78 \mathrm{nA}$ to $1.68 \mathrm{nA}$ (figure 5). These experiments were performed on the same day that identical recordings failed to detect hIK1 in keratinocytes treated for 3 days with calcium and VitD. The fact that all ras-transformed NIH3T3 cells tested responded with large magnitude mIK1 currents is consistent with previous reports from several groups, including our own, concerning these cells $[24,25]$.

mRNA levels for mIK1 and hIK1 were measured from cell cultures made in parallel to those used for electrophysiology. hIK1 levels in keratinocytes treated for 5 days with calcium and VitD were approximately half of those for mIK1 in ras-transformed NIH3T3 cells. This level of hIK1 expression was entirely consistent with the initial expression studies shown in figure 1. By comparison, channel mRNA levels in wild type NIH3T3 and untreated keratinocytes were considerably lower. It is important to note, however, that despite the 19-fold difference in mIK1 mRNA levels between wild type $(0.41 \%)$ and ras-transformed $(7.97 \%)$ NIH3T3 cells, wild type cells still display readily observable whole cell mIK1 currents at approximately $10 \%$ of the amplitude of ras-transformed cells $[24,25]$. Further, the mIK1 mRNA level in wild type 3T3 cells is exceeded by hIK1 mRNA levels in keratinocytes from the following treatment groups: VitD for 5 days; VitD and calcium for 3 days; VitD and calcium for 5 days. Therefore, although IK currents can be observed in 3T3 cells expressing mIK1 mRNA at approximately $0.4 \%$ of GAPDH, no IK currents were observed in any of the 19 

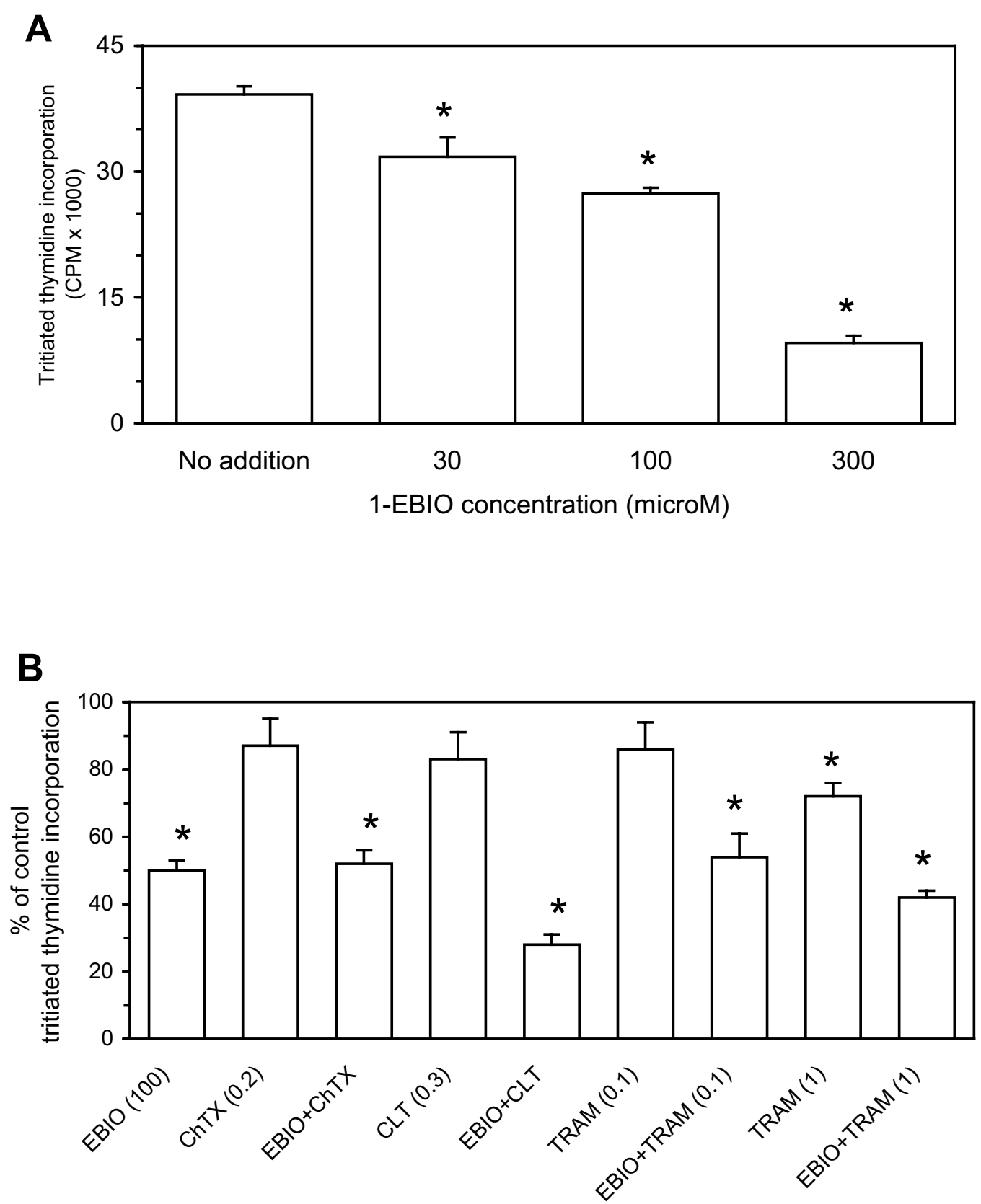

Figure 4

Inhibition of keratinocyte mitogenesis by the hIKI agonist I-EBIO is not reversed by hIKI blockers charybdotoxin (ChTX), clotrimazole (CLT) and TRAM-34 (TRAM). Keratinocyte mitogenesis was assessed by measuring tritiated-thymidine incorporation over a 24 hour period. (A) single experiment, representative of four total, showing dosedependence of I-EBIO inhibition of mitogenic activity. Mitogenic inhibition was significant $(*)$ at the concentrations of I-EBIO tested (ANOVA, 95\% confidence interval). (B) Cumulative data showing failure of hIKI blockers ChTX, clotrimazole, and TRAM-34 to reverse the inhibitory effect of $100 \mu \mathrm{M}$ I-EBIO on keratinocyte mitogenesis. Percent difference in thymidine incorporation versus untreated control cells plotted with each column representing mean \pm SEM of 3-4 experiments. Numbers next to drugs indicate $\mu \mathrm{M}$ concentrations. I00 $\mu \mathrm{M}$ I-EBIO, with or without hIKI blockers present, caused significant reductions in thymidine incorporation relative to untreated control cells (indicated by asterisks). That is, blockers did not reverse inhibition due to I-EBIO which would be expected if I-EBIO was acting via agonism of hIKI. TRAM-34 itself caused significant proliferative inhibition but only at I $\mu$ M. 

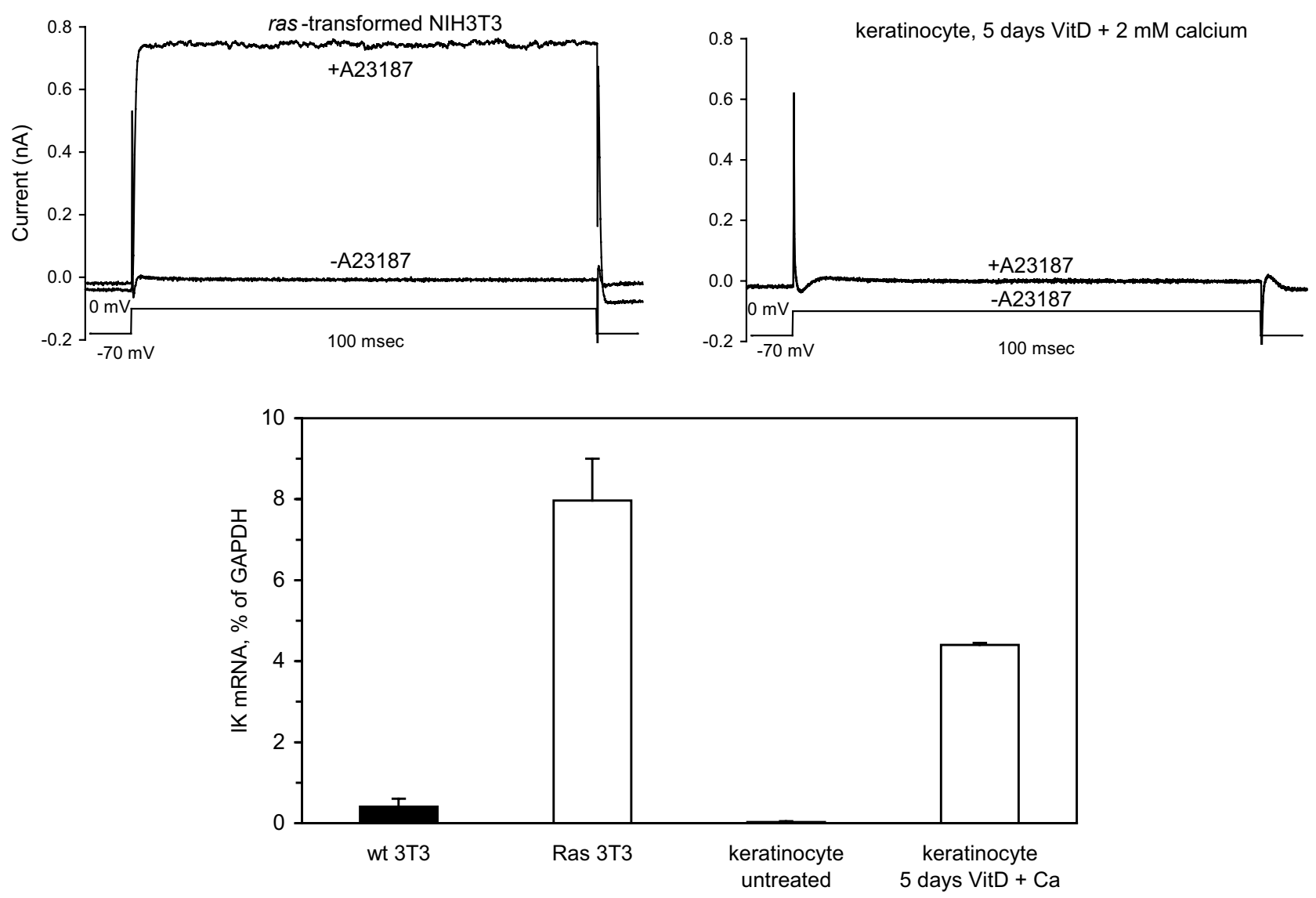

\section{Figure 5}

Both NIH3T3 cells and calcium/VitD-treated keratinocytes have abundant IKI mRNA but only NIH3T3 display IKI currents. Top panels show whole cell patch clamp recordings from a keratinocyte (treated 5 days with 2 mM calcium and I $\mu \mathrm{M}$ VitD) and ras-transformed NIH3T3 fibroblast. Each panel comprises records taken prior to and then during I2 minute application of $10 \mu \mathrm{M} \mathrm{A23} \mathrm{I87,} \mathrm{a} \mathrm{calcium} \mathrm{ionophore} \mathrm{which} \mathrm{allows} \mathrm{IK} \mathrm{channel} \mathrm{activity} \mathrm{to} \mathrm{be} \mathrm{observed} \mathrm{during} \mathrm{voltage}$ steps to $0 \mathrm{mV}$ (voltage protocol shown beneath current records). mIKI current is readily observed in ras-transformed NIH3T3 cells in response to A23 I87, while hIKI activity is absent from keratinocytes. Bottom panel shows real-time PCR analysis of IK mRNA levels from sibling cultures to those used for the patch clamp recordings (mIKI for NIH3T3, hIKI for keratinocytes). Each column represents mean \pm SEM of two cultures. For comparison filled columns show mRNA levels for wild type (wt, non-transformed) NIH3T3 cells and untreated keratinocytes ( $0.06 \mathrm{mM}$ calcium, no VitD).

keratinocytes from cultures which express hIK1 mRNA at 0.7 to $4.4 \%$ of GAPDH.

\section{Why are no functional hIKI channels observed despite the fact that hIKI mRNA is abundant and appears to be physiologically regulated?}

Our results showing 1-EBIO inhibits keratinocyte proliferation suggest an involvement of hIK1 in this process, but the hIK1 blocker ChTX failed to reverse this inhibition. 1EBIO has also been shown to modulate cell growth in the HaCaT keratinocyte cell line, but 1 -EBIO and other benzimidazolones also affect chloride (and other) conductances $[26,27]$. which are known to be present in HaCaT $[9,28]$. Although the presence of chloride channels in primary keratinocytes has not been addressed, it is possible that 1-EBIO affects proliferation in these cells and HaCaT through channels other than hIK1, or through some target yet to be determined. In addition, the electrophysiological effects of 1-EBIO and indeed the presence of hIK1 currents in HaCaT cells have not been subjected to direct voltage clamp analysis $[9,10]$. , therefore, a critical electrophysiological comparison of these cells with pri- 


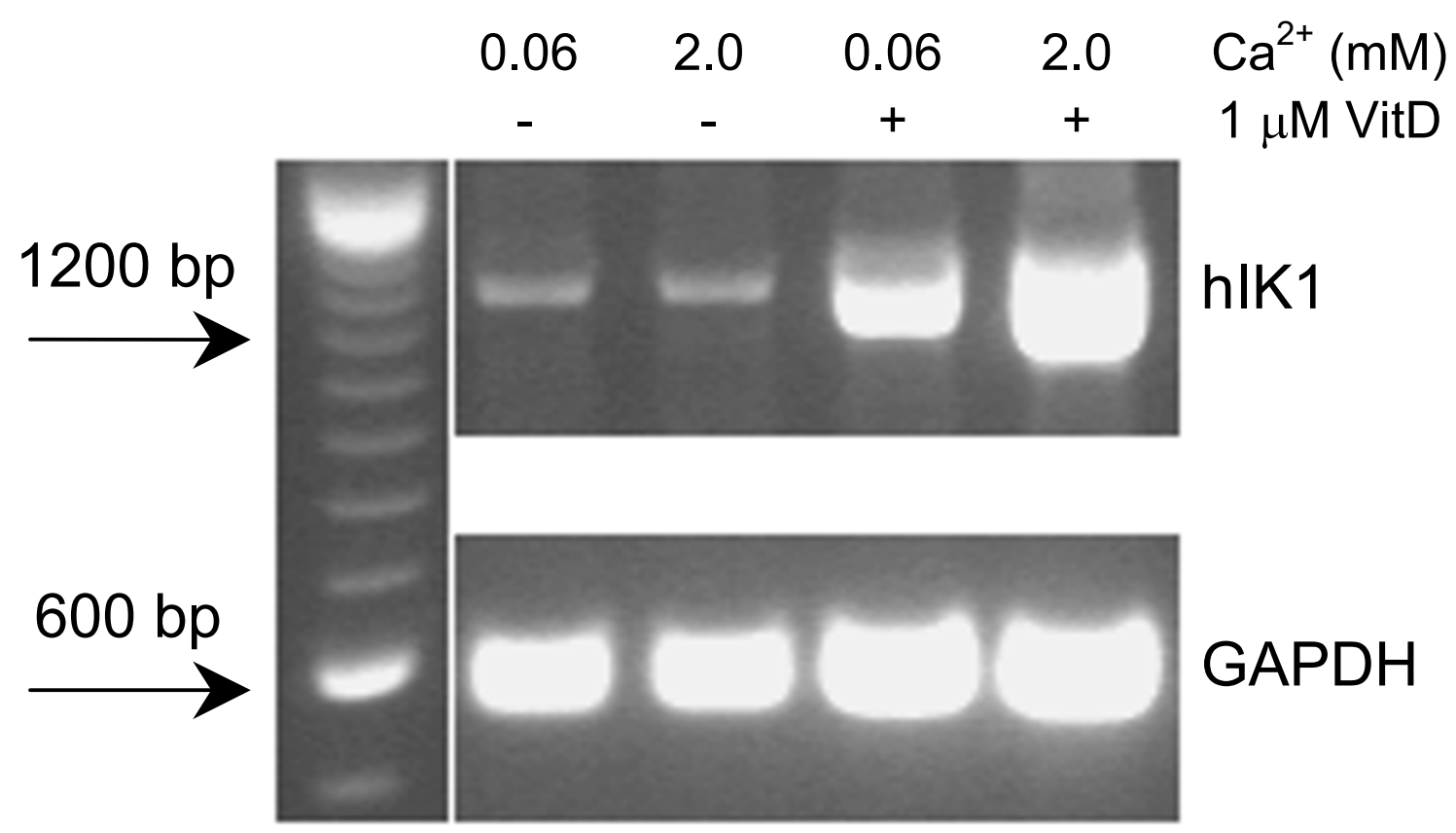

\section{Figure 6}

End-point PCR for the full-length protein coding sequence of hIKI confirms real-time PCR results. Conventional end-point RT-PCR amplifying the coding sequence (I 28I bp of I284) of hIKI was performed on keratinocytes grown for five days in either proliferative $(0.06 \mathrm{mM}$ calcium $)$ or anti-proliferative, pro-differentiating $(2 \mathrm{mM}$ calcium, I $\mu M$ VitD) culture conditions. GAPDH, 615 bp of the complete coding sequence, is shown as a loading control. Full-length hIKI message is upregulated by VitD and more so by combining VitD with calcium, exactly as for the real-time PCR results (see figure I).

mary keratinocytes is not yet possible. Thus, it appears that although hIK1 is expressed at the mRNA level in primary human keratinocytes as well as skin, functional channels are not formed, and thus cell growth studies utilizing hIK1 pharmacophores must be interpreted with caution.

At present we have no mechanistic explanation for why primary keratinocytes express hIK1 mRNA but lack any apparent functional channels. One purely technical possibility is that channels are produced but require cellular conditions for activity that we could not reproduce during the patch clamp experiments. However, IK channels are quite functionally robust and all reported recording conditions are straightforward and virtually identical, even in cells as diverse as fibroblasts, T-cells, microglia, and smooth muscle. A more interesting possibility is that hIK1 channels are produced in keratinocytes but they localize to organellar membranes and not the plasma membrane.
This hypothetical intracellular hIK1 would not be detected with conventional patch clamp techniques, and it could be both sensitive to lipophilic compounds like 1EBIO while being protected from peptide toxins (e.g., ChTX). Despite this intriguing possibility a physiological function for intracellularly localized IK channels is also uncertain. Another possibility is that our TaqMan based PCR was detecting a truncated [29] or splice variant hIK1 [30] which is electrophysiologically non-functional. To address this possibility, the hIK1 mRNA upregulation experiment shown in figure 1 was repeated, using endpoint PCR for the full-length hIK1 sequence instead of the real-time method (which relies on detection of less than $100 \mathrm{bp}$ out of the coding sequence). Figure 6 shows that exposure to calcium or VitD for 5 days significantly increases levels of full-length hIK1 mRNA. Further, commercial sequencing (SeqWright Inc., Houston, TX) of the PCR products showed the base content to be $>90 \%$ identical to the reported hIK1 sequence, the non-matching 
$10 \%$ being due to sequencing ambiguities and incomplete sequencing of the $3^{\prime}$ and $5^{\prime}$ ends. Most importantly, sequencing showed no evidence for alternative first exons as has been reported for the closely related SK3 channel to encode non-functional, dominant negative variants [30,31]. Finally, similar to our results with hIK1, calciumregulated expression of mRNA for certain P2Y purinergic receptor subtypes in human keratinocytes has been reported coincident with a lack of functional receptors [32].

\section{Conclusions}

The critical role for calcium influx in keratinocyte proliferation and differentiation strongly indicates an electrophysiological component to these processes, and hIK1 has been modeled to serve an essential role in controlling calcium mobilization in other non-neuronal, non-muscle cell types. However, our results suggest that the functional electrophysiology of primary human keratinocytes, and possibly intact human skin, does not include hIK1. Further, the lack of functional hIK1 channels suggests caution must be used when interpreting results with IK1 pharmacophores in human epidermal keratinocyte cells and skin.

\section{Abbreviations}

ChTX, charybdotoxin; 1-EBIO, 1-ethyl-2-benzimidazolinone; GAPDH, glyceraldehyde-3-phosphate dehydrogenase; IK, intermediate-conductance calcium-activated potassium channel; INV, involucrin; TRAM-34, 1- [(2chlorophenyl)diphenylmethyl]-1H-pyrazole; VitD, 1,25$(\mathrm{OH})_{2}-\mathrm{D}_{3}$; TG-1, transglutaminase-1.

\section{Competing Interests}

None declared.

\section{Authors' Contributions}

SGR conceived of the study and with SR and MK directed its design and coordination. VM did the PCR analyses, and $A B, W Q$ and SGR performed the cell proliferation and patch clamp experiments. SGR wrote the manuscript with significant input from $A B, S R$ and VM. All authors read and approved the final manuscript.

\section{Acknowledgements}

An NSF grant (MCB-0080796) partially supported this research.

\section{References}

I. Cahalan MD, Wulff H, Chandy KG: Molecular properties and physiological roles of ion channels in the immune system.J Clin Immunol 200I, 2 I:235-252.

2. SG Rane: Potassium channels as targets for modulating cell growth and differentiation. In Perspectives in Drug Discovery and Design Volume 15//6. Edited by: Darbon H, Sabatier J-M. Dordrecht, Netherlands: Kluwer Academic; 1999:295-3II.

3. Lu X, Fein A, Feinstein MB, O'Rourke FA: Antisense knock out of the inositol 1,3,4,5-tetrakisphosphate receptor GAP IIP4BP in the human erythroleukemia cell line leads to the appearance of intermediate conductance $\mathrm{KCa}$ channels that hyperpolar- ize the membrane and enhance calcium influx. J Gen Physiol 1999, II 3:81-96.

4. Devor DC, Singh AK, Bridges RJ, Frizzell RA: Modulation of $\mathrm{Cl}^{-}$ secretion by benzimidazolones. II. Coordinate regulation of apical $\mathbf{G}_{\mathrm{Cl}}$ and basolateral $\mathbf{G}_{\mathrm{K}}$. Am J Physiol 1996, 27 I:L785-L795.

5. Devor DC, Singh AK, Frizzell RA, Bridges RJ: Modulation of $\mathbf{C l}$ secretion by benzimidazolones. I. Direct activation of a $\mathrm{Ca}^{2+}$ dependent K+ channel. Am J Physiol 1996, 27 I:L775-L784.

6. Jensen BS, Strobaek D, Olesen SP, Christophersen P: The $\mathbf{C a}^{2+}$-activated $\mathrm{K}^{+}$channel of intermediate conductance: a molecular target for novel treatments? Curr Drug Targets 200I, 2:40I-422.

7. Vazquez E, Nobles M, Valverde MA: Defective regulatory volume decrease in human cystic fibrosis tracheal cells because of altered regulation of intermediate conductance $\mathrm{Ca}^{2+}$ dependent potassium channels. Proc Natl Acad Sci U S A 200I, 98:5329-5334.

8. Dotto GP: Signal transduction pathways controlling the switch between keratinocyte growth and differentiation. Crit Rev Oral Biol Med 1999, 10:442-457.

9. Koegel $\mathrm{H}$, Alzheimer $\mathrm{C}$ : Expression and biological significance of $\mathrm{Ca}^{2+}$-activated ion channels in human keratinocytes. FASEB J 200I, I 5: I 45-I54.

10. Koegel H, Kaesler S, Burgstahler R, Werner S, Alzheimer C: Unexpected down-regulation of the hIKI $\mathrm{Ca}^{2+}$-activated $\mathrm{K}^{+}$channel by its opener I-ethyl-2-benzimidazolinone in $\mathrm{HaCaT}$ keratinocytes. J Biol Chem 2003, 278:3323-3330.

II. Mauro T, Dixon DB, Komuves L, Hanley K, Pappone PA: Keratinocyte $\mathrm{K}^{+}$channels mediate $\mathrm{Ca}^{2+}$-induced differentiation. J Invest Dermatol 1997, 108:864-870.

12. Mauro TM, Isseroff RR, Lasarow R, Pappone PA: Ion channels are linked to differentiation in keratinocytes. J Membr Biol 1993, 132:201-209.

13. Rane SG: The growth regulatory fibroblast IK channel is the prominent electrophysiological feature of rat prostatic cancer cells. Biochem Biophys Res Commun 2000, 269:457-463.

14. Peña TL, Chen SH, Konieczny SF, Rane SG: Ras/MEK/ERK Up-regulation of the fibroblast $K_{\mathrm{Ca}_{\mathrm{a}}}$ channel FIK is a common mechanism for basic fibroblast growth factor and transforming growth factor- $\beta$ suppression of myogenesis. J Biol Chem 2000, 275: 13677-13682.

15. Rane SG: $\mathbf{A} \mathbf{C a}^{2+}$-activated $\mathbf{K}^{+}$current in ras-transformed fibroblasts is absent from nontransformed cells. Am J Physiol 1991, 260: C104-CII2.

16. Takahashi $H$, Ibe $M$, Kinouchi $M$, Ishida-Yamamoto $A$, Hashimoto $Y$, lizuka $\mathrm{H}$ : Similarly potent action of I,25-dihydroxyvitamin D3 and its analogues, tacalcitol, calcipotriol, and maxacalcitol on normal human keratinocyte proliferation and differentiation. J Dermatol Sci 2003, 3 I:2I-28.

17. Verstuyf A, Segaert S, Verlinden L, Bouillon R, Mathieu C: Recent developments in the use of vitamin $D$ analogues. Expert Opin Investig Drugs 2000, 9:443-455.

18. Bollinger-Bollag W, Bollag RJ: I,25-Dihydroxyvitamin $D_{3}$, phospholipase $D$ and protein kinase $C$ in keratinocyte differentiation. Mol Cell Endocrinol 200I, 177:173-182.

19. Varani J, Fligiel SE, Schuger L, Perone P, Inman D, Griffiths CE, Voorhees J]: Effects of all-trans retinoic acid and $\mathrm{Ca}^{++}$on human skin in organ culture. Am J Pathol 1993, I 42:189-198.

20. Jensen BS, Strobaek D, Christophersen P, Jorgensen TD, Hansen C, Silahtaroglu A, Olesen SP, Ahring PK: Characterization of the cloned human intermediate-conductance $\mathrm{Ca}^{2+}$-activated $\mathrm{K}^{+}$ channel. Am J Physiol 1998, 275:C848-C856.

21. Syme CA, Gerlach AC, Singh AK, Devor DC: Pharmacological activation of cloned intermediate- and small-conductance $\mathrm{Ca}^{2+}$-activated $\mathrm{K}^{+}$channels. Am J Physiol Cell Physiol 2000, 278:C570-C58I.

22. Wulff H, Miller MJ, Hansel W, Grissmer S, Cahalan MD, Chandy KG: Design of a potent and selective inhibitor of the intermediate-conductance $\mathrm{Ca}^{2+}$-activated $\mathrm{K}^{+}$channel, IKCa I: a potential immunosuppressant. Proc Natl Acad Sci U S A 2000, 97:8I5I-8I56.

23. Vandorpe DH, Shmukler BE, Jiang L, Lim B, Maylie J, Adelman JP, de Franceschi L, Cappellini MD, Brugnara C, Alper SL: cDNA cloning and functional characterization of the mouse $\mathrm{Ca}^{2+}$-gated $\mathrm{K}^{+}$ channel, mIKI. Roles in regulatory volume decrease and erythroid differentiation. J Biol Chem 1998, 273:2 I542-21553. 
24. Draheim HJ, Repp H, Dreyer F: Src-transformation of mouse fibroblasts induces a $\mathrm{Ca}^{2+}$-activated $\mathrm{K}^{+}$, current without changing the T-type $\mathbf{C a}^{2+}$ current. Biochim Biophys Acta 1995, I 269:57-63.

25. Huang Y, Rane SG: Potassium channel induction by the Ras/Raf signal transduction cascade. J Biol Chem 1994, 269:3 I I83-3 I I89.

26. Cuthbert AW: Assessment of CFTR chloride channel openers in intact normal and cystic fibrosis murine epithelia. $\mathrm{Br} J$ Pharmacol 200I, I 32:659-668.

27. Singh S, Syme CA, Singh AK, Devor DC, Bridges RJ: Benzimidazolone activators of chloride secretion: potential therapeutics for cystic fibrosis and chronic obstructive pulmonary disease. J Pharmacol Exp Ther 2001, 296:600-6II.

28. Wohlrab D, Wohlrab J, Markwardt F: Electrophysiological characterization of human keratinocytes using the patch-clamp technique. Exp Dermatol 2000, 9:219-223.

29. Syme CA, Hamilton KL, Jones HM, Gerlach AC, Giltinan L, Papworth GD, Watkins SC, Bradbury NA, Devor DC: Trafficking of the $\mathrm{Ca}^{2+}$-activated $\mathrm{K}^{+}$channel, hIKI, is dependent upon a C-terminal leucine zipper. J Biol Chem 2003, 278:8476-8486.

30. Kolski-Andreaco AA, Tomita H, Shakkottai VG, Gutman GA, Cahalan MD, Gargus JJ, Chandy KG: SK3-IC: a dominant-negative suppressor of $\mathbf{S} \mathbf{K}_{\mathbf{C}}$ and $\mathbf{I K}_{\mathbf{C a}}$ channels. J Biol Chem 2004, 279:6893-6904.

31. Tomita H, Shakkottai VG, Gutman GA, Sun G, Bunney WE, Cahalan MD, Chandy KG, Gargus JJ: Novel truncated isoform of SK3 potassium channel is a potent dominant-negative regulator of SK currents: implications in schizophrenia. Mol Psychiatry 2003, 8:524-535.

32. Burrell HE, Bowler WB, Gallagher JA, Sharpe GR: Human keratinocytes express multiple $P 2 Y$-receptors: evidence for functional $\mathbf{P} 2 \mathbf{Y}_{1}, \mathbf{P} 2 \mathbf{Y}_{2}$, and $\mathbf{P} 2 \mathbf{Y}_{4}$ receptors. J Invest Dermatol 2003, I 20:440-447.

\section{Pre-publication history}

The pre-publication history for this paper can be accessed here:

http://www.biomedcentral.com/1471-5945/4/7/prepub

\section{Publish with Bio Med Central and every scientist can read your work free of charge}

"BioMed Central will be the most significant development for disseminating the results of biomedical research in our lifetime. "

Sir Paul Nurse, Cancer Research UK

Your research papers will be:

- available free of charge to the entire biomedical community

- peer reviewed and published immediately upon acceptance

- cited in PubMed and archived on PubMed Central

- yours - you keep the copyright

Submit your manuscript here:

http://www.biomedcentral.com/info/publishing_adv.asp
BioMedcentral 